\title{
Fluorescence lifetime imaging ophthalmoscopy: autofluorescence imaging and beyond
}

\author{
Lydia Sauer $^{1} \cdot$ Alexandra S. Vitale ${ }^{1}$ Natalie K. Modersitzki ${ }^{1}$ Paul S. Bernstein (i) ${ }^{1}$
}

Received: 14 October 2020 / Revised: 20 October 2020 / Accepted: 4 November 2020 / Published online: 2 December 2020

(c) The Author(s), under exclusive licence to The Royal College of Ophthalmologists 2020

\begin{abstract}
Fluorescence lifetime imaging ophthalmoscopy, FLIO, has gained large interest in the scientific community in the recent years. It is a noninvasive imaging modality that has been shown to provide additional information to conventional imaging modalities. The FLIO device is based on a Heidelberg Engineering Spectralis system. Autofluorescence lifetimes are excited at $473 \mathrm{~nm}$ and recorded in two spectral wavelength channels, a short spectral channel (SSC, 498-560 nm) and a long spectral channel (LSC, 560-720 nm). Typically, mean autofluorescence lifetimes in a $30^{\circ}$ retinal field are investigated. FLIO shows a clear benefit for imaging different retinal diseases. For example, in age-related macular degeneration (AMD), ring patterns of prolonged FLIO lifetimes 1.5-3.0 mm from the fovea can be appreciated. Macular telangiectasia type 2 (MacTel) shows a different pattern, with prolonged FLIO lifetimes within the typical MacTel zone. In Stargardt disease, retinal flecks can be appreciated even before they are visible with other imaging modalities. Early hydroxychloroquine toxicity appears to be detectable with FLIO. This technique has more potential that has yet to be discovered. This review article focuses on current knowledge as well as pitfalls of this technology. It highlights clinical benefits of FLIO imaging in different ophthalmic and systemic diseases, and provides an outlook with perspectives from the authors.
\end{abstract}

\section{Introduction}

Retinal imaging provides an opportunity to detail and study ocular pathology with a new approach. Imaging modalities are constantly expanded to discover novel ways to precisely image the eye. Recently, there has been a growing interest in imaging modalities that explore not only structural but also metabolic changes in the eye, as these may manifest prior to structural damage. One technique known to show metabolic changes in tissues is autofluorescence lifetime imaging. Autofluorescence lifetimes describe the fluorescence decay over time without the application of fluorescent dye and are unique features of individual substances [1]. Autofluorescence lifetimes may change with alterations in the microenvironment or the molecular composition.

Paul S. Bernstein

paul.bernstein@hsc.utah.edu

1 Department of Ophthalmology and Visual Sciences, Moran Eye Center, University of Utah School of Medicine, 65 Mario Capecchi Drive, Salt Lake City, UT 84132, USA
Previously, fluorescence lifetime imaging has been used in the diagnosis of various cancers as well as atherosclerotic disease [2]. Gastrointestinal and bronchial cancers showed differences in autofluorescence lifetimes of the tumour versus healthy tissue. Oral carcinomas exhibited faster decays compared to normal tissue, which was attributed to varying ratios of free and bound $\mathrm{NAD}(\mathrm{P}) \mathrm{H}$ [3]. Even brain tumours, such as gliomas, have altered lifetimes compared to healthy tissue. Multiple skin diseases have also been studied with lifetime imaging, and individual types of atherosclerotic plaques may show different fluorescence lifetimes [2].

One of the most interesting approaches to autofluorescence lifetime imaging is in the ophthalmoscopic realm. Fluorescence lifetime imaging ophthalmoscopy (FLIO) dates back to the early 2000s when Professors Schweitzer and Hammer from the University Hospital of Jena, Germany, first showed that it is possible to detect autofluorescence lifetimes from the human retina in vivo [4]. The researchers built an experimental device and were able to show images of fluorescence lifetimes in healthy eyes and eyes with age-related macular degeneration (AMD), the latter with prolonged FLIO lifetimes [5]. In the following years, the FLIO technology was further developed by Heidelberg Engineering, and first prototypes were used in 2012 in a clinical setting in Jena (Germany), 
Bern (Switzerland) and Ilmenau (Germany). These centres focused on establishing scientific as well as clinical knowledge of this technology. Reproducible patterns were found for different diseases, and retinal changes in FLIO lifetimes were associated with changes in early disease stages. The technology was expanded and is now available to more research centres, including Salt Lake City (USA) and Lübeck (Germany). Many studies showed the value of FLIO as more than just a research device, and ongoing research will likely determine even more areas where FLIO proves to be useful. Overall, this field is rapidly evolving, and this review article aims to give an overview of the current knowledge in this field.

\section{Technological aspects}

Fluorescence lifetime imaging has a long history in the analysis of metabolic changes in cell cultures. As the fluorescence spectra from many different fluorophores in biological tissue overlap, fluorescence lifetime imaging provides an additional parameter to provide contrast. Furthermore, fluorescence lifetimes are independent of fluorescence intensity, fluorophore concentration, and quantum yield. Despite these advantages, the technique is highly complex, especially when investigating fluorescence lifetimes in vivo. Metabolic as well as structural factors can influence fluorescence lifetimes. In cell cultures, individual lifetimes can be attributed to specific components, but the in vivo analysis is challenging, as fluorescence lifetimes represent a collection of different fluorophores rather than a single component or fluorophore.

Nevertheless, applications in vivo show promising findings, and fluorescence lifetime imaging has been used in cancer diagnosis and skin evaluations [2], but one of the most promising applications is within ophthalmology. A clinical Spectralis-based prototype device manufactured by Heidelberg Engineering (Heidelberg, Germany) has been available in the past decade. This system records fundus autofluorescence lifetimes by utilizing time-correlated, single-photon counting (TCSPC) [6]. The system is equipped with a $473 \mathrm{~nm}$ wavelength pulsed diode laser emitting pulses with a frequency of $80 \mathrm{MHz}$ at a full width at half maximum (FWHM) of 89 picoseconds (ps). Laser safety has been evaluated in previous works [7]. In brief, the single pulse peak power was calculated to be $28 \mathrm{~mW}$, focused as an $\sim 75 \mu^{2}$ spot on the retina, resulting in an intensity of 37 $\mathrm{kW} / \mathrm{cm}^{2}$. Applied onto a single spot with a continuous wave laser, this laser power would result in retinal damage. However, FLIO uses a pulsed laser with a low duty cycle for the laser power density, and the scanning procedure allows only a few laser pulses to sum up at an individual spot, resulting in a mean power much lower than critical values [7]. Based on regulations from the American National Standards Institute (ANSI) Z136.1-2000 [8], FLIO fulfils all safety regulations for a class 1 laser, and the pulse energy is almost a factor of $10^{4}$ smaller compared to the acceptable exposure limit [9].

The Heidelberg FLIO images an area of 256 by 256 pixels, corresponding to a 30 by $30^{\circ}(9$ by $9 \mathrm{~mm}$ ) field at the retina, which can be centred at the fovea or any other area of the retina. Two sensitive hybrid photon-counting detectors (HPM-100-40, Becker\&Hickl GmbH, Berlin, Germany) record fluorescence photons from each pixel in 1024 time channels, and two separate photon arrival histograms are thereby obtained for each pixel (one histogram per spectral channel). Photon arrival histograms are mathematically approximated to calculate the fluorescence lifetime.

The Heidelberg Engineering Spectralis FLIO device detects fluorescence decays in two separate spectral wavelength channels, a short spectral channel (SSC; 498 to $560 \mathrm{~nm}$ ) and a long spectral channel (LSC; 560 to $720 \mathrm{~nm}$ ). This separation was based on a publication that suggested that the LSC predominantly records information from lipofuscin, while the SSC records a large variety of other fluorophores, including reduced nicotinamide adenine dinucleotide, flavin adenine dinucleotide and retinal carotenoids $[5,10]$. It would be interesting to obtain FLIO images from other wavelengths ranges, but the two wavelength channels in current FLIO instruments have proven to be very helpful in the clinical setting, as different diseases manifest more significantly in one of the two channels and can therefore be distinguished. Furthermore, the SSC is somewhat affected by the lens fluorescence, whereas the LSC is relatively unaffected by this.

The Heidelberg FLIO system utilizes a high-contrast confocal infra-red reflectance image to account for eye movements during the measurement. Thereby, each fluorescence photon is recorded at the corresponding spatial retinal location. To obtain FLIO images in good quality, a minimal signal threshold of $\sim 1000$ photons per pixel should be achieved, resulting in a measurement time of $\sim 2$ min per eye.

To analyse the fluorescence decays obtained with FLIO, the software SPCImage (Becker\&Hickl GmbH, Berlin, Germany) has mostly been used. SPCImage traditionally is used in fluorescence lifetime imaging microscopy (FLIM), but a similar software is available for in vivo ophthalmic imaging. Typically, the fluorescence decay is approximated using a two- or three-exponential approach according to:

$$
\frac{I(\mathrm{t})}{I_{0}}=\operatorname{IRF} \otimes \sum_{i} \alpha_{i} * e^{-\frac{t}{\tau_{i}}}
$$

In this equation, $I(\mathrm{t})$ represents the fluorescence intensity at time $\mathrm{t}, I_{0}$ the maximal fluorescence intensity, IRF the 
instrument response function, $\alpha$ the amplitude, and $\tau$ each lifetime component. $\otimes$ represents the convolution integral with the IRF. With this calculation, two or three different lifetimes are obtained $\left(\tau_{1}, \tau_{2}, \tau_{3}\right)$ depending on the model used (bi- or tri-exponential approach). The measure of interest is the amplitude-weighted, mean autofluorescence lifetime $\left(\tau_{\mathrm{m}}\right)$. In a tri-exponential setting, $\tau_{\mathrm{m}}$ can be calculated as follows:

$\tau_{\mathrm{m}}=\frac{\alpha_{1} \times \tau_{1}+\alpha_{2} \times \tau_{2}+\alpha_{3} \times \tau_{3}}{\alpha_{1}+\alpha_{2}+\alpha_{3}}$.

The fluorescence does not decay completely within the time of the next laser impulse from the scanning laser ophthalmoscope (12.5 ns), so SPCImage accounts for this incomplete decay. Most clinical FLIO studies also use a sliding average pixel kernel (squared binning) of either $3 \times 3$ or $5 \times 5$ pixels to reduce noise and improve quality of the fit.

One two-channel FLIO measurement acquires two autofluorescence intensity images, two FLIO lifetime images, and a corresponding infra-red reflectance image. Figure 1 gives an example of measurements obtained with FLIO for a healthy eye.

Although FLIO lifetimes can be investigated within individual pixels, in the clinical setting FLIO lifetimes are often averaged across areas of interest. SPCImage provides a masking tool where manual masks can be drawn onto any area of interest. This can be done on the FLIO image as well as on the corresponding autofluorescence intensity image. The software provides mean FLIO lifetimes found across all pixels within the masked area and allows for a quantitative analysis. In clinical studies, the utilization of standardized grids has also been very helpful. The Early Treatment Diabetic Retinopathy Study (ETDRS) grid has typically been used. Two additional programmes are available that apply the ETDRS grid onto the FLIO lifetime image in a similar fashion as the masking in SPCImage. These two programmes are the FLIO-Reader (ARTORG Center for Biomedical Engineering Research, University of Bern, Switzerland) and FLIMX (Institute for Biomedical Engineering and Informatics, Technische Universität Ilmenau, Ilmenau, Germany). We hope that a single software will be available in the future that can apply standardized grids as well as individual masks onto FLIO images. The ETDRS grid is shown in Fig. 1.

FLIO images represent pseudo-colour maps of individual lifetimes. When investigating or presenting these images, it is important to understand that the heat map can be adjusted in order to highlight certain details. This adjustment does not change the FLIO lifetimes and is comparable to image processing for CT imaging of the chest, where contrast adjustments lead to a better visualization of either the bones, the soft tissue, or the lung. In a similar way, FLIO lifetime maps may be adjusted to show different disease-related features. As FLIO lifetimes prolong with increasing age, contrast adjustments may improve image quality [11].

FLIO imaging is highly reproducible, and previous studies quantified the inter-individual variability of FLIO by the coefficient of variation (CV). The first study investigating the reproducibility of FLIO was published in 2013 when Klemm et al. performed FLIO imaging on ten healthy volunteers with ten replicate images per volunteer. The fovea, optic disc and papillo-macular bundle were investigated regarding reproducibility, and foveal $\mathrm{CVs}$ of 7.9\% (SSC) and $17.7 \%$ (LSC) were reported for dilated eyes [12]. A second study was published by Dysli et al. [13], where 31 phakic healthy volunteers (62 eyes) were imaged twice ( $>1 \mathrm{~h}$ apart) with non-dilated pupils and then again after pupil dilation. The authors investigated different ETDRS grid areas and reported highly reproducible FLIO lifetimes. The CVs were $17 \%$ (SSC) and 11\% (LSC) for the central area, $12 \%$ (SSC) and 7\% (LSC) for the inner ring, and 9\% (SSC) and 6\% (LSC) for the outer ring [13]. Good repeatability in mydriasis was again reported by Kwon et al. [14] with CVs of 9-16\%. Furthermore, right and left eyes showed good correlations for both spectral channels [13]. No correlation was found between FLIO lifetimes and refractive errors for either spectral channel in a cohort of healthy patients with mean spherical equivalents around $-1.9 \pm 2.6$ dioptres [14].

Pupil dilation plays an important role in FLIO imaging. Dysli et al. [13] showed that the correlation between age and a prolongation of FLIO lifetimes was more pronounced in non-dilated pupils. They attributed this finding to a larger lens impact in non-dilated measurements. In 2019, Kwon and colleagues investigated 63 eyes from 32 healthy, phakic subjects and concluded that without dilation, FLIO lifetimes are longer in the SSC. In addition, image acquisition takes longer without pupil dilation. All FLIO studies, except those investing the effect of mydriasis, were performed with maximal pupil dilation.

Bleaching effects with FLIO have been studied in 21 healthy volunteers [15]. Three individual measurements were taken, all in full mydriasis. The first measurement was obtained after $30 \mathrm{~min}$ of dark adaptation, the second image followed a 1-min light bleaching at a luminance of $3200 \mathrm{~cd} / \mathrm{m}^{2}$, and the third measurement followed another 10 -min period of dark adaptation. Acquisition times in the bleached state were significantly shorter in bleached (78 s) versus dark-adapted states (136 s before bleaching, $138 \mathrm{~s}$ after second dark adaptation). The number of photons collected and mean FLIO lifetimes were similar in all three measurements. Minor but significant prolongations with bleaching were reported for the LSC. These changes were less than $10 \%$ and were within the inter-individual variability of FLIO [12]. Therefore, the authors 

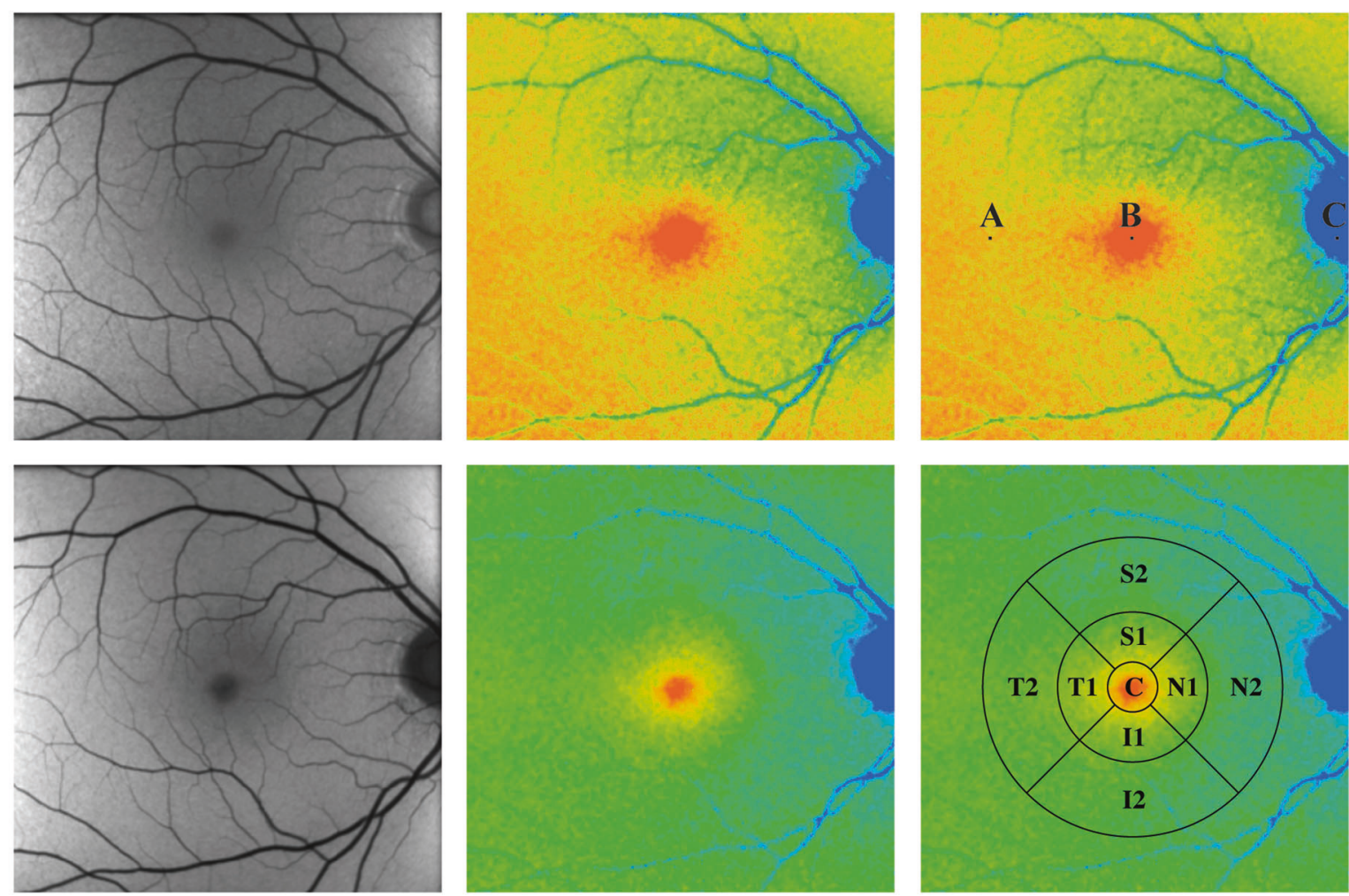

A

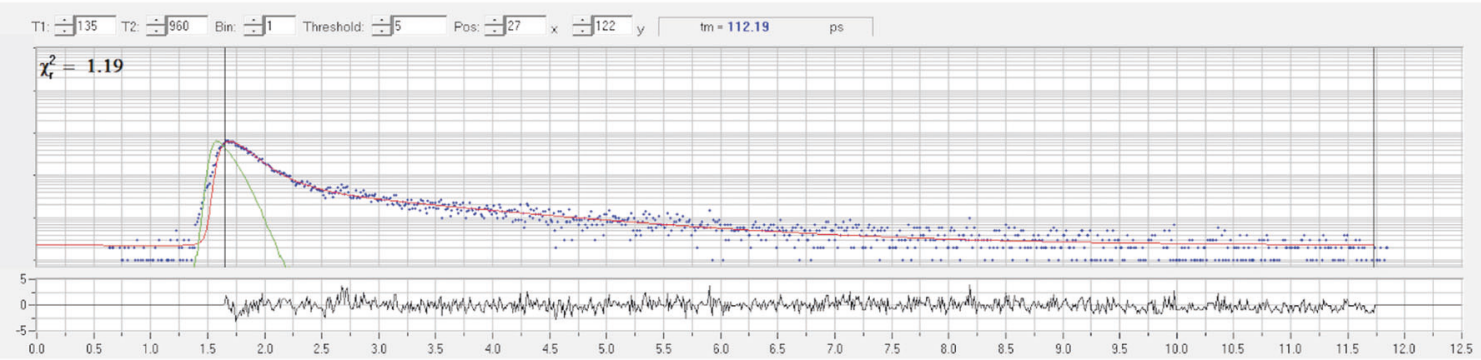

B

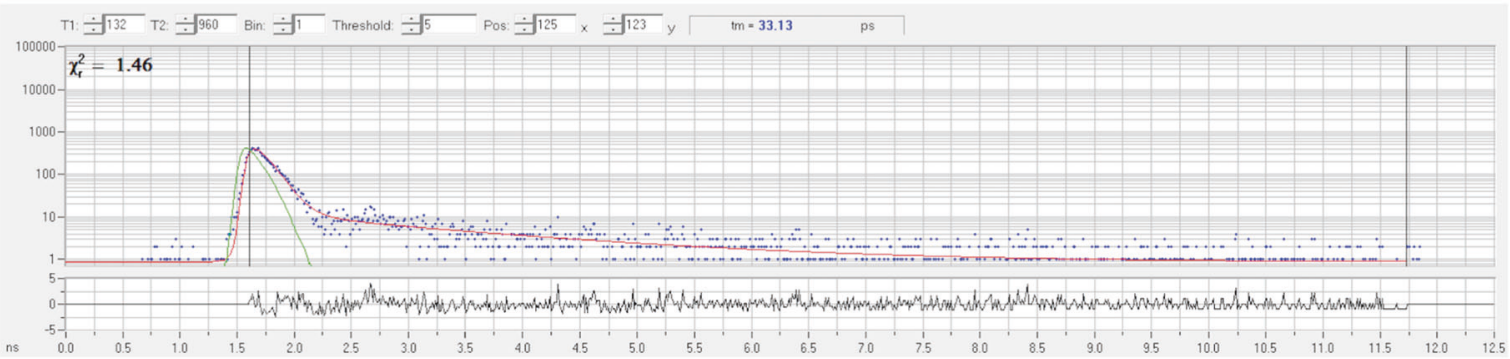

C

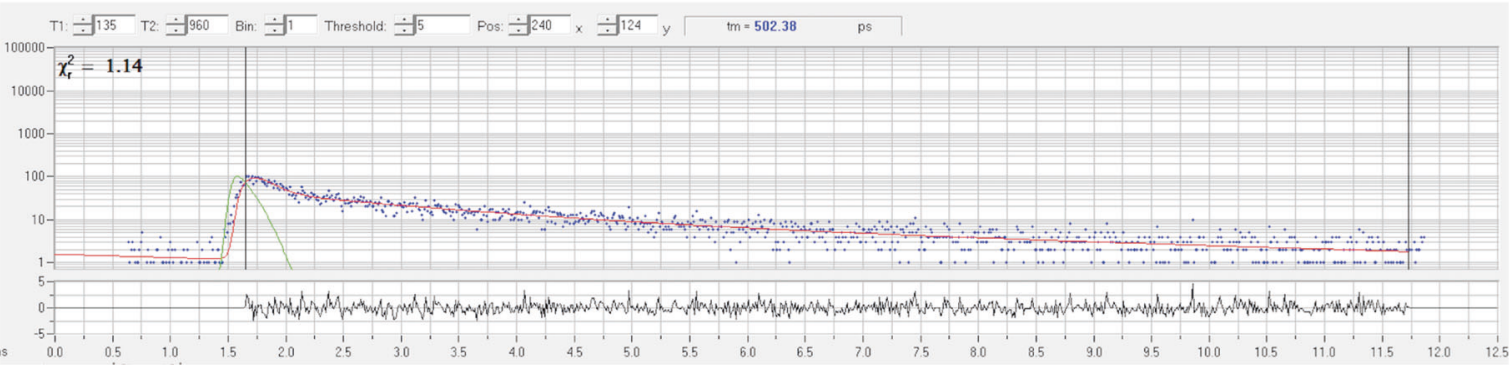


Fig. 1 FLIO in a healthy eye. FAF intensity and FLIO lifetime images from a healthy eye. Short (SSC, 498-560 nm) and long (LSC, 560-720 nm) spectral channels are shown. A-C Individual pixels with the decay curves shown below. The standardized ETDRS grid is shown as well.

concluded that bleaching does not confound clinical FLIO imaging.

Lifetime imaging studies of certain parts of the eye or the fundus typically use the terms fundus autofluorescence lifetimes, autofluorescence decays, autofluorescence lifetimes, or FLIO lifetimes (typically abbreviated as FLTS) when reporting autofluorescence lifetimes. In the past, however, fundus autofluorescence lifetimes have also been abbreviated with the term $F A F$ lifetimes, which is confusing due to the common abbreviation $F A F$ for fundus autofluorescence (intensity) imaging. Autofluorescence lifetimes are intensity-independent. It is therefore important to distinguish between conventional autofluorescence intensity imaging and FLIO, and we would like to encourage the usage of the terms FLIO lifetimes or FLTS. In contrast to intensity, which would typically be higher or lower, it may make sense to use the words prolonged and shortened for FLIO lifetimes. Both short and long lifetimes can be seen in areas with high as well as low FAF intensity. Furthermore, it is important not to refer to lifetimes by colours. As mentioned above, the colour scaling is an arbitrary measure that helps to highlight differences in FLIO lifetimes and can be adjusted depending on the goals of the study. Changing the colour range will not change the lifetimes, but it is important to report the lifetime range along with the colour scale because FLIO lifetimes never become more red or more blue.

\section{Clinical aspects}

\section{General knowledge and healthy eyes}

In the clinical setting, FLIO has been used to investigate a variety of pathological conditions as well as healthy eyes. Healthy eyes have been analysed in great detail by multiple centres [7, 10, 11, 13, 14]. The fundus of healthy eyes was found to have a consistent FLT pattern, which is shown in Fig. 1. The longest lifetimes were observed in the area of the optic disc, and early FLIO studies suggested this to be the influence of collagen and connective tissue [4, 5, 16]. Across the retina, intermediate lifetimes can be found that likely originate from the retinal pigment epithelium (RPE) and are potentially caused by lipofuscin. This conclusion was also drawn from a study investigating eyes with macular holes [17], as here FLIO lifetimes were obtained directly from the RPE without overlying retinal layers.
FLTs within holes were not significantly different from those found across the retina. The foveal centre shows shortest FLIO lifetimes, which were found to be influenced by the carotenoids of the macular pigment (MP).

MP was initially believed to only absorb light but to not emit fluorescence. In 2001, it was found that MP shows fluorescence in the context of Raman imaging [18]. In 2015, FLIO studies showed a strong correlation of MP with foveal FLIO lifetimes (SSC: $r=-0.76$ LSC: $r=-0.66, p<0.001$ for both) [7]. Further studies confirmed this finding [7, 10]. Retinal carotenoids themselves also show short FLIO lifetimes when investigated in a quartz cuvette, and FLTs of about 50-60 ps were reported [10]. Supplementation with retinal carotenoids leads to a significant shortening of FLIO lifetimes in as little as 3 months. If carotenoid supplementation is discontinued, FLIO lifetimes prolong to the baseline within 3 months [19]. In patients with albinism, who do not have MP, short FLIO lifetimes in the foveal centre are absent [10].

FLIO lifetimes also show an age dependency. In 2014, Dysli et al. [13] investigated 31 healthy, phakic eyes and found that FLTs prolong with increasing age. The correlation of FLTs with age was stronger if pupils were not dilated [13]. Differences regarding mydriasis are discussed in the Technological Aspects section. To a large extent, the prolongation of FLIO lifetimes with age is believed to be caused by the aging natural lens, but a recent study found that some prolongations of FLIO lifetimes with increasing age may be lens-independent [11]. Ninety-seven eyes from 97 healthy subjects were included, within an age range from 5 to 85 years, and the two spectral channels did not change in the same way with increasing age. The SSC showed significant changes with age starting around age 35, whereas the LSC showed prolongations of FLIO lifetimes from childhood on. While pseudophakic patients had shorter FLIO lifetimes in the SSC, the LSC seemed to be somewhat more independent of the lens status, and FLIO lifetimes in pseudophakic patients were not significantly shorter compared to age-matched healthy subjects with natural lenses. This may arise from the increasing amount of lipofuscin with increasing age detected in the LSC. The accumulation of lipofuscin with age has been speculated to increase autofluorescence intensity in quantitative autofluorescence imaging [20], and it is likely that the prolongations of FLIO lifetimes with increasing age in the LSC are due to lipofuscin.

FLIO lifetimes are independent of gender, as was shown by investigating 97 eyes from 97 healthy subjects [11]. The same study did not find any differences based on race; however, the study was limited due to a small number of black participants ( 8 subjects). The authors found only a non-significant trend of longer lifetimes in the SSC in darker pigmented individuals. Furthermore, long FLIO 
lifetimes were reported for melanin that was measured ex vivo in a quartz cuvette (SSC $1400 \mathrm{ps,} \mathrm{LSC} 850$ ps). This study should be repeated with a more diverse population.

Multiple studies investigated the effects of retinal thickness on FLTs. The first study investigated the inner nasal area of the ETDRS grid and found significant correlations of retinal thickness with FLIO lifetimes as well as the amount of detected photons [13]. A second study investigated the central subfield retinal thickness and found that simply correlating the central subfield thickness with FLIO lifetimes showed that shorter lifetimes were found in eyes with a thinner central retina [7], but those eyes also showed larger amounts of MP. When correcting for MP, no correlation of FLTs with retinal thickness was found [7]. A third study investigated retinal thickness and FLIO lifetimes in areas outside of the fovea and found significant correlations of retinal thickness with FLIO lifetimes in both spectral channels [14]. The same study also investigated vascular density and FLIO lifetimes; a weak correlation of vascular density and FLTs was found in the LSC $(r=-0.119, p<0.05)$, but no significant correlation in the SSC [14].

\section{Posterior segment imaging}

Most clinical FLIO studies have focused on diseases of the posterior segment, particularly retinal diseases. Figure 2 shows how different retinal diseases manifest in FLIO imaging. This section highlights the current FLIO knowledge in some retinal diseases. As research advances, more diseases will likely be investigated.

\section{Age-related macular degeneration (AMD)}

AMD is a frequent cause of blindness in the elderly population, but despite its common presentation, treatment options are still lacking [21-23]. AMD has been classified as either exudative or non-exudative, both forms leading to irreversible vision loss and retinal atrophy in its end stages [24]. Supplementing retinal carotenoids in the AREDS2 study was found to slow disease progression [25]. Although the AREDS2 study included only patients with intermediate AMD, it is likely that all forms of AMD benefit from carotenoid supplementation. It would be especially interesting if disease progression could be slowed even before damage of the disease manifests clinically.

FLIO imaging shows a distinct and characteristic pattern in eyes with AMD, a ring-shaped prolongation of the LSC FLIO lifetimes in an area of about $1.50-3.0 \mathrm{~mm}$ from the fovea [26]. Figure 3 depicts this pattern. Compared to healthy eyes, the area of the pattern (outer ring of the ETDRS grid) was found to be significantly longer in AMD compared to age-matched healthy eyes. FLIO lifetimes from the outer ring were $545 \mathrm{ps}$ in phakic AMD compared to $370 \mathrm{ps}$ in phakic healthy eyes, $(p<0.001)$, and $475 \mathrm{ps}$ (AMD) compared to $419 \mathrm{ps}$ (healthy), $(p<0.05)$, in pseudophakic eyes [27]. Another study found similar differences; lifetimes in the outer ring were between 55 and $80 \mathrm{ps}$ longer in eyes with AMD compared to healthy eyes [26]. Central FLIO lifetimes did not show significant differences, which indicates that the outer ring is most affected by these FLT changes. Eyes with early disease stages as well as increased risk due to prior familial presentation were also found to show this pattern in many cases. Furthermore, it was reported that eyes with advanced disease stages show significantly longer lifetimes in the area of the outer ring compared to earlier disease stages $(p<0.05)$, whereas the fovea showed similar FLIO lifetimes [26]. This indicates that the FLIO pattern may advance with disease progression.

The origin of the AMD-associated FLIO pattern is still unclear, but it has been speculated that this prolongation of FLIO lifetimes may reflect an accumulation of bis-retinoids or sub-RPE deposits. Vascular alterations are less likely but could potentially also account for changes in the lifetimes. Nevertheless, the pattern is a unique and consistent finding in eyes with AMD and should be further explored.

Eyes with geographic atrophy related to late stage AMD show extremely prolonged FLTs, but occasionally short lifetimes can be found in the area of atrophy [28, 29]. Another pathological hallmark in AMD is drusen, extracellular debris deposits that localize between the inner collagenous layer of Bruch's membrane and the basal lamina of the RPE $[22,30]$. In addition, pigment epithelial detachments (PEDs) which can be drusenoid, fibro-vascular, serous, or haemorrhagic, often represent the initial step of vision loss [31]. Various studies have investigated drusen and PEDs [26, 27, 32-34]. One study investigated drusen ex vivo and found that drusen with the same fluorescence spectrum can show different lifetimes [33]. The same study reported that lifetimes of drusen were longer compared to lifetimes from surrounding tissue in in vivo human eyes as well as RPE in ex vivo samples [33]. Further studies again found the heterogeneity of FLIO lifetimes from drusen, and while some studies reported longer lifetimes in drusen compared to surrounding tissue [26], other studies did not find these differences [27]. This discrepancy may be caused by different study populations included in these studies, as the study that found significant prolongations of drusen included only eyes with non-exudative AMD. A study investigating PEDs gave further information on the difference in drusen lifetimes, as drusenoid PEDs showed long FLIO lifetimes, whereas serous or haemorrhagic PEDs showed short FLIO lifetimes [32]. FLIO may not be the best modality to detect drusen, as drusen do not exhibit uniform FLIO lifetimes. On the contrary, FLIO may 


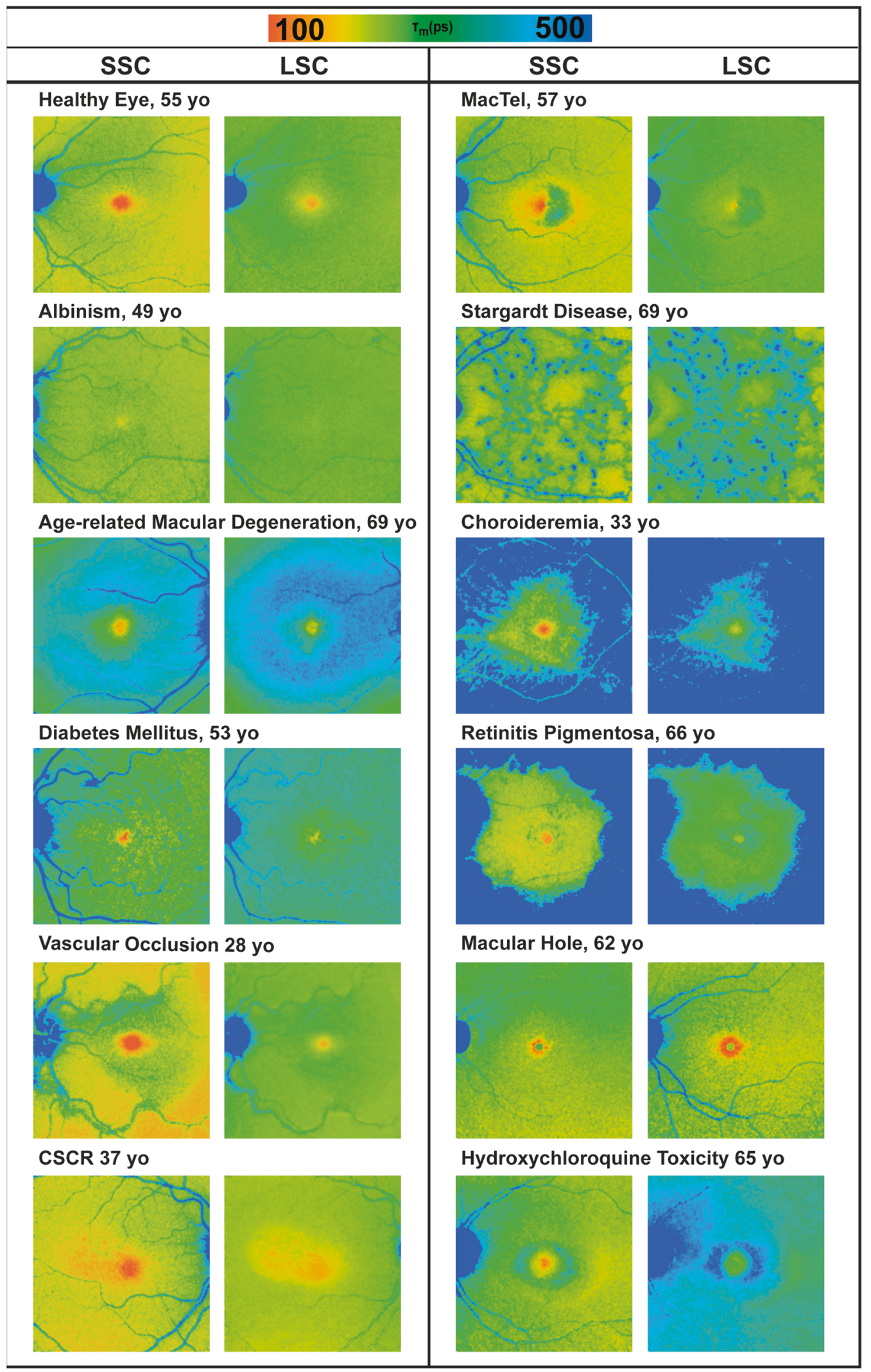

Fig. 2 FLIO in different retinal diseases. FLIO lifetime images from short (SSC, 498-560 nm) and long (LSC, 560-720 nm) spectral channels of different retinal diseases. 
Fig. 3 FLIO in AMD. FAF intensity and FLIO lifetime images from an eye with AMD. Short (SSC, 498-560 nm) and long (LSC, 560-720 nm) spectral channels are shown.

\section{FLIO Lifetime FAF Intensity}

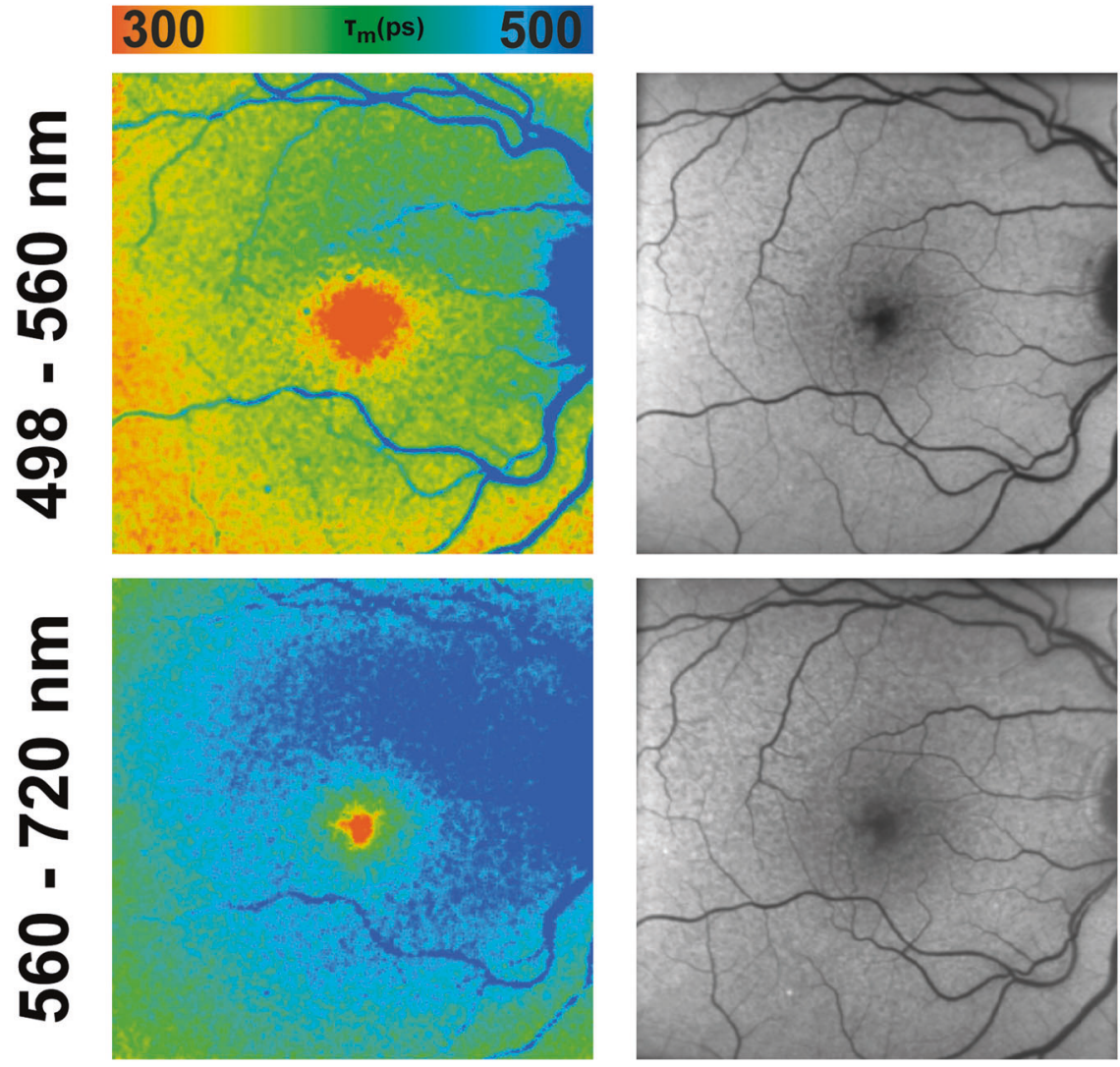

be a great modality to further understand various drusen pathologies, metabolism, and phenotypes based on the differences in FLIO lifetimes. Overall, FLIO in eyes with AMD shows very promising findings, and there is a large potential for FLIO to become an indispensable tool to evaluate AMD in the future.

\section{Diabetes mellitus}

Diabetes often affects the eye and leads to visual disturbances. Common ocular complications include microangiopathy, disturbances in the blood-retina barrier, increased inflammation, retinal degeneration, and macular oedema [35-37]. In addition, hyperglycaemia leads to protein glycation and to the formation of advanced glycation endproducts (AGEs), which were shown to fluoresce [38]. FLIO imaging has been studied in diabetic patients, both with and without diabetic retinopathy [16, 39-41]. FLTs were prolonged in diabetes, which was thought to be due to reduced oxidative metabolism and an increase of AGEs [41]. An accumulation of AGEs in the crystalline lens may contribute to this [41]. A detailed study was conducted on an early experimental device, where 48 patients with type 2 diabetes but no clinical signs of diabetic retinopathy were investigated [40]. Although the retinas of these patients appeared healthy, FLIO imaging already showed significant prolongations of FLTs in these eyes. Another study investigated 33 patients with nonproliferative diabetic retinopathy compared to 28 controls. Again, prolonged retinal FLIO lifetimes were reported. Interestingly, the lenses of patients with diabetes showed shorter FLIO lifetimes compared to controls [39]. These studies highlight the potential metabolic alterations that can be seen with FLIO even before structural damage manifests and may be helpful in monitoring the retina of patients with diabetes.

\section{Vascular occlusions}

Vascular occlusions in the retina lead to sudden, typically non-painful vision loss. In the case of retinal artery occlusion, the central retinal artery can either be completely occluded (central retinal artery occlusion: CRAO), or a branch of the central retinal artery can occlude (branch retinal artery occlusion: BRAO). Ophthalmoscopically, opacification due to swelling of inner retinal layers is 
observed in the acute phase. In the post-acute phase, this swelling typically resolves within $2-3$ weeks, with restored retinal transparency. Vision loss is often permanent due to inner retinal atrophy, optic nerve head pallor, and permanent vascular changes within affected areas.

The first study that used FLIO to investigate vascular occlusions was done with an early experimental FLIO device. Two eyes were investigated, and a prolongation of FLIO lifetimes was found [42]. A second FLIO-based study included 24 patients with retinal artery occlusions, 13 with CRAO (11 acute and four post-acute; 2 patients were examined in both stages) and 11 with BRAO (seven acute and four post-acute). Mean FLIO lifetimes were prolonged in both spectral channels in the acute stage. Significant prolongations were found in the inner and outer ring of the ETDRS grid, with no significant prolongation in the central area. In addition, no significant correlations were found between retinal thickness and FLIO lifetimes. Compared to the control eye, FLIO lifetimes from the inner ring of eyes with CRAO were prolonged by 59\% (209 ps) in the SSC and by $22 \%$ ( $89 \mathrm{ps}$ ) in the LSC. No significant differences were found when comparing the post-acute stage to control eyes despite atrophy of inner retinal layers, which may indicate the small contribution of inner retinal layers to the total FLIO lifetimes. Eyes with BRAO showed similar findings. Nevertheless, other studies showed that the contribution of neuronal retinal layers should not be neglected when investigating FLIO [43]. In a mouse model, prolonged lifetimes were found in eyes with ischaemia [44]. The exact mechanisms are not quite understood, but the prolonged FLIO lifetimes may indicate metabolic alterations and unbalanced intracellular ionic concentrations [45].

\section{Central serous chorioretinopathy (CSCR)}

CSCR is a common retinal disease characterized by deterioration of the RPE, resulting in serous RPE detachments and RPE atrophy [46]. Multimodal imaging displays focal leakage in fluorescence angiography, mottled atrophic hyperfluorescence in FAF, and hyporeflectance corresponding to areas of subretinal fluid or PEDs seen in OCT imaging [47]. One study investigated fluorescence lifetime changes in 35 CSCR patients. Results showed that patients with chronic CSCR or areas with current or previous PEDs or subretinal fluid had significantly shorter fluorescence lifetimes in both spectral channels [48]. While increased duration of disease was associated with prolonged fluorescence lifetimes, acute stages displayed shortened fluorescence lifetimes, even in areas where subretinal fluid was absent. In addition, patients with retinal pigment atrophy associated with previous PEDs or subretinal fluid showed prolonged FLIO lifetimes in those respective areas as well as poor visual acuity [48].

\section{Macular Telangiectasia type 2 (MacTel)}

MacTel is a disease with complex inheritance that causes bilateral central visual impairment [49]. It was believed to manifest in the 4-6th decade of life, but there have been recent reports of MacTel in younger individuals. A phase 3 clinical trial investigating the effects of ciliary neurotrophic factor on MacTel to slow or even stop disease progression is currently in progress, which emphasizes the importance of accurate characterization of affected individuals at early disease stages [50]. FLIO shows retinal disease-related changes very early, sometimes even before there is structural damage and before changes are visible with other imaging methods [51-53]. For example, a 26year-old female with multiple first-degree family members affected with MacTel whose macula appeared clinically normal had characteristic MacTel changes when imaged with FLIO [51].

In FLIO, MacTel presents with a typical pattern of prolonged FLTs in the so-called MacTel zone, an oval area $9^{\circ}$ horizontal and $5^{\circ}$ vertical centred at the fovea. This area appears to harbour most MacTel-related pathology. FLIO images show a temporal crescent or a ring of prolonged FLIO lifetimes in this area [52]. Figure 4 shows this pattern. The inner temporal area (T1) from the ETDRS grid showed prolonged FLTs in MacTel (382 ps) compared to healthy eyes $(298 \mathrm{ps})$ in the SSC $(p<0.001)$. A second study showed that this pattern is very reproducible and found longer FLIO lifetimes in patients with less MP. MP distribution is altered in eyes with MacTel, and instead of a central peak, a ring-like enhancement of MP outside of the MacTel zone can be found. FLIO also shows a ring of short FLTs surrounding the MacTel zone, but prolonged FLTs inside the MacTel zone cannot be explained by the loss of MP alone.

One study investigated children of MacTel probands and found changes that may be indicative and predictive of disease development [53]. Follow-up studies of these family members will give further insights into whether FLIO will have the ability to detect MacTel potentially years before the onset of structural changes. Finally, some studies have investigated longitudinal changes in eyes with MacTel. One study included four patients and found a prolongation of $22 \%$ in 2.1 years [54]. A second study investigated 33 patients with MacTel and found an annual progression of $9 \mathrm{ps}$ in the SSC and $8 \mathrm{ps}$ in the LSC for the temporal area.

\section{Stargardt disease}

Stargardt disease, an inherited retinal disease that often manifests in childhood, causes a retinal dystrophy and can result in severe vision loss [55-57]. The disease itself is 
Fig. 4 FLIO in MacTel. FAF intensity and FLIO lifetime images from an eye with MacTel. Short (SSC, 498-560 $\mathrm{nm}$ ) and long (LSC, 560-720 nm) spectral channels are shown.

\section{FLIO Lifetime FAF Intensity}

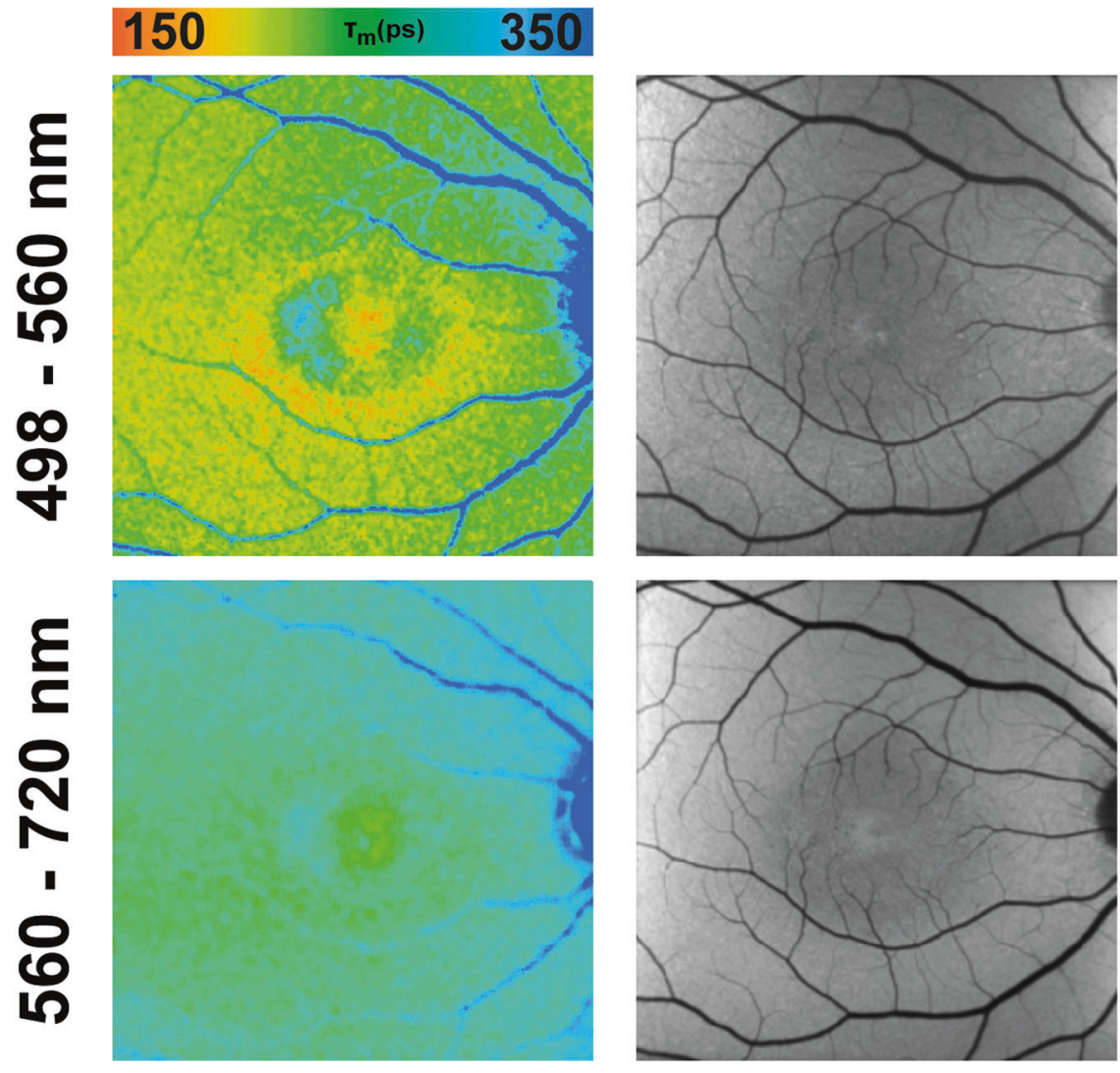

caused by mutations in the $A B C A 4$ gene, encoding for an ATP-binding cassette protein that transports N-retinylidenephosphatidylethanolamine across photoreceptor disc membranes [57]. The mutation leads to an accumulation of bisretinoids, all-trans retinal, lipofuscin and other visual cycle by-products [58-60]. In the early stages, FAF intensity is typically increased, while photoreceptor function remains normal [60]. Later in the course of the disease, yellow spots, so-called retinal flecks, can appear across the fundus, eventually leading to retinal degeneration. Sixteen patients with Stargardt disease were initially investigated with FLIO [61]. The retina without flecks, deposits, or atrophy showed FLIO lifetimes around $322 \mathrm{ps,} \mathrm{which} \mathrm{were} \mathrm{comparable} \mathrm{to}$ age-matched controls. Retinal flecks showed shortened (242 ps) as well as prolonged (474 ps) FLTs. Flecks with short FLIO lifetimes are believed to be new onset flecks, and follow-up examinations reveal a shift towards longer FLTs over time [62]. Figure 5 shows this finding. Short lifetimes may represent different compounds of the degenerating photoreceptor cells, possibly retinaldehyde adducts and bis-retinoids. Longer FLIO lifetimes in older flecks were mostly attributed to lipofuscin and A2E. The short lifetime flecks were visible with FLIO even before they could be appreciated in conventional autofluorescence imaging. With various pharmaceutical trials in progress to reduce the accumulation of by-products from the visual cycle and to slow the development of atrophy [63, 64], FLIO imaging may be extremely helpful to highlight progression of the disease.

\section{Choroideremia}

Choroideremia is an inherited retinal dystrophy caused by a mutation in the CHM gene, which encodes for Rab escort protein 1 , and results in degeneration of the RPE, photoreceptor, and choroidal layers [65]. Choroideremia has been previously investigated with FLIO imaging by Dylsi and coworkers, describing short fluorescence lifetimes in areas of MP and preserved RPE islands, and prolonged lifetimes in areas of diseased and atrophic retina [65]. The most recent study by Vitale et al. [66] reported similar findings but noted heterogeneous mixtures of short and long FLIO lifetimes in both atrophic and preserved retinal areas. Upon further examination, it was found that short lifetimes in atrophic areas could be attributed to the presence of outer retinal tubulations (ORTs) [66]. In addition, short fluorescence lifetimes in retinal blood vessels were observed in all 15 choroideremia patients. Follow-up analysis of 
Fig. 5 FLIO in Stargardt disease. FAF intensity and FLIO lifetime images from an eye with Stargardt disease. Short (SSC, 498-560 nm) and long (LSC, $560-720 \mathrm{~nm}$ ) spectral channels are shown.

\section{FLIO Lifetime FAF Intensity}

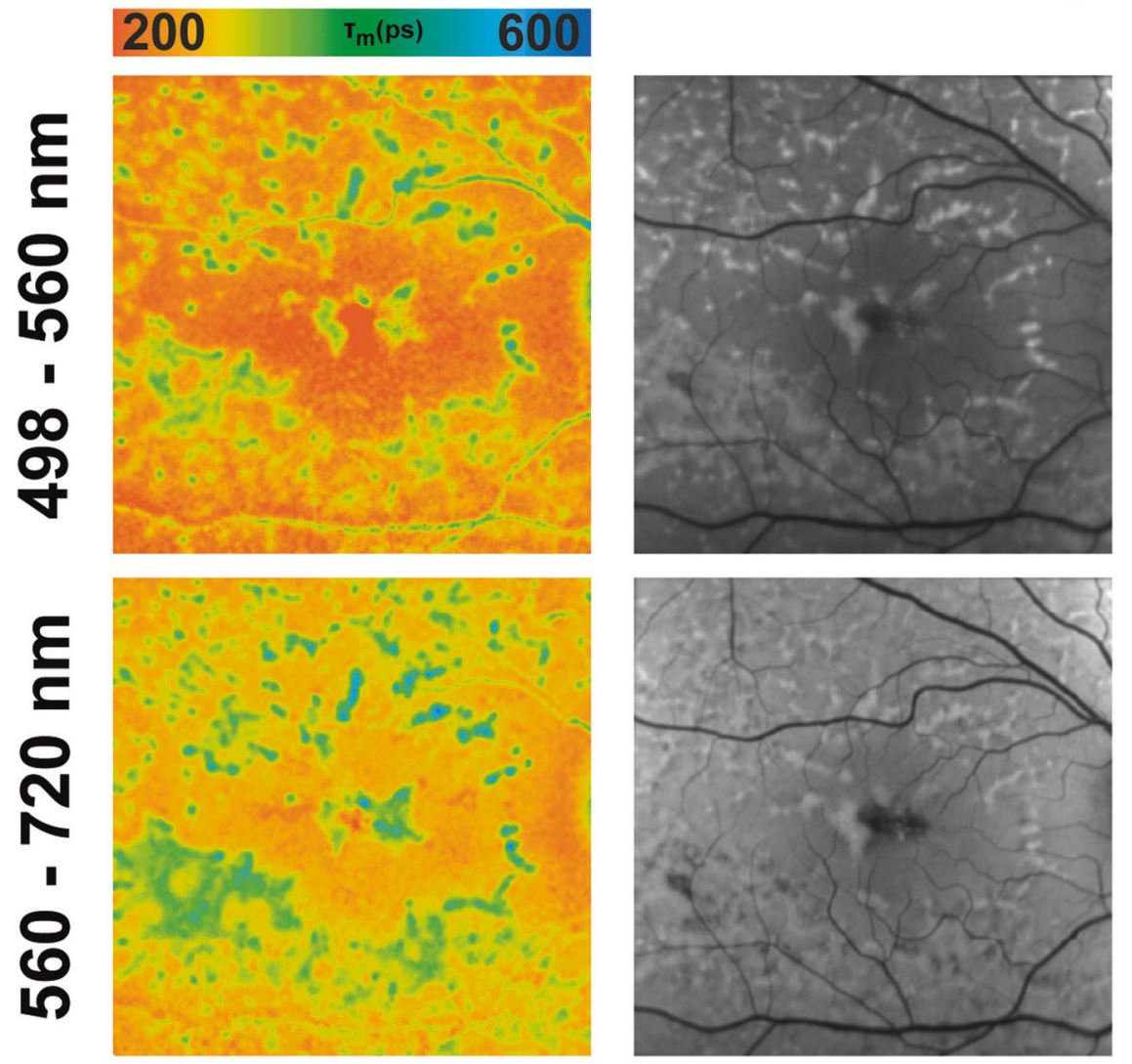

three patients showed that compared to baseline imaging, significant prolongation of lifetimes were observed in corresponding areas of extended atrophy. Interestingly, one follow-up patient showed an increase of short lifetimes due to an accumulation of ORTs in the parafoveal area, indicating that they have some influence in altering FLIO lifetimes [66]. While all patients were genetically confirmed for choroideremia, there were no observable genotype-phenotype correlations. As gene therapy trials for choroideremia are in progress, FLIO may help identify functional areas, disease-related risk, and subsequent progression.

\section{Retinitis pigmentosa (RP)}

$\mathrm{RP}$ is a group of inherited degenerative diseases that lead to night blindness, visual field constriction, peripheral vision loss, and eventual legal blindness [67]. RP has a large genetic heterogeneity, including autosomal dominant, autosomal recessive, and X-linked inheritance, and is one of the most common causes of legal blindness. Patients with RP typically show retinal vessel attenuation, bone spicules in the peripheral retina, and retinal thinning and atrophy. Two studies have investigated FLIO findings in patients with RP [68, 69]. Fluorescence lifetimes in areas of photoreceptor atrophy alone were slightly prolonged, and combined photoreceptor and RPE atrophy showed even further prolonged FLIO lifetimes. FLIO can identify photoreceptor and RPE atrophy, as well as preserved photoreceptor segments within large areas of RPE atrophy. In addition, FLIO can be used to pinpoint ellipsoid zone loss that correlates with retinal function. Typically, the central macula remains relatively spared from RP and is comparable to healthy controls. Surrounding this region, ringshaped patterns both in autofluorescence intensity as well as in FLIO lifetimes were observed. These were found to be the most common in patients with autosomal dominant RP inheritance [69]. Rings of short FLIO lifetimes in the LSC may indicate different genetic or pathophysiologic processes. The distinct FLIO pattern may offer a deeper understanding into the disease progression in eyes affected with RP.

\section{Macular holes and retinal detachments}

Full-thickness idiopathic macular holes and symptomatic vitreomacular adhesion have a prevalence of $0.02-0.8 \%$ and cause visual disability [70]. FLIO lifetimes were 
investigated in 37 patients with full-thickness idiopathic macular holes [17]. It was observed that short central FLIO lifetimes were disrupted and that together with the inner retinal layers they were displaced adjacent to the defect, likely due to relocation of the foveal MP. Macular holes often show an operculum, a small opacity at the posterior vitreous above the defect. This opacity may contain MP and has correspondingly short FLIO lifetimes. Fifteen patients were followed after surgery, and in 11 cases, the surgery was successful. Central FLIO lifetimes correlated significantly with best visual acuity, and shorter lifetimes in the central fovea were found in eyes with better visual acuity. This may be an important finding, as surgical outcomes vary.

Analysis of rhegmatogenous retinal detachments before and after surgery showed similar results. Fifty-eight patients were included, and all had macula-off rhegmatogenous retinal detachments, which were successfully repaired with surgery [71]. Previously detached retinal areas showed significantly prolonged FLIO lifetimes, while short FLIO lifetimes in the centre of the ETDRS grid correlated with better visual acuity. FLIO may therefore be useful in predicting long-term surgical outcomes.

\section{Hydroxychloroquine toxicity}

Hydroxychloroquine, also known as Plaquenil, is a drug that is commonly used in the treatment of rheumatoid arthritis and systemic lupus erythematosus. For many patients, hydroxychloroquine is the only effective treatment option; however, irreversible retinal toxicity and vision loss can be a side effect of this medication after prolonged use. With a half-life of more than 40 days [72], it was found that retinal damage continues to worsen up to 6 months after the drug is discontinued [73]. Therefore, early detection and correct interpretation of retinal changes due to hydroxychloroquine are of utmost importance. Diagnosing retinal toxicity related to hydroxychloroquine can be easy in some cases with advanced atrophy but often is more challenging in cases of early toxicity $[74,75]$. Multifocal ERG as well as OCT imaging give good indications on the state of the retina; however, if a patient has multiple retinal diseases, it may be difficult to distinguish hydroxychloroquine toxicity from other retinal abnormalities.

FLIO has been shown to be of help in diagnosing retinal toxicity at an early stage, possibly before the onset of structural changes. Two studies were published that investigated hydroxychloroquine toxicity with FLIO [76, 77]. In both studies, patients with known hydroxychloroquine retinopathy as well as patients on hydroxychloroquine without known retinopathy were evaluated. One study investigated 12 patients [76], and the other study included seven patients with toxicity [77]. Both studies showed similar results of prolonged FLIO lifetimes in the inner ring of the ETDRS grid, the presumed area of toxicity (about $400 \mathrm{ps}$ and $375 \mathrm{ps}$, respectively) compared to the healthy retina (about $250 \mathrm{ps}$ and $313 \mathrm{ps,} \mathrm{respectively,} p<0.001$ for both studies). Prolongations were found in both spectral channels but were found predominantly in the SSC. Figure 6 shows typical FLIO findings. Furthermore, abnormal FLTs with a ring of prolonged FLIO lifetimes were found in eyes with very early toxicity. Both studies also found significantly altered FLIO lifetimes in patients on hydroxychloroquine without retinal toxicity. It will be interesting to continue to follow these patients closely, as most of the patients with abnormalities only in FLIO continued to use the drug. Patients on hydroxychloroquine with other retinal changes unrelated to hydroxychloroquine toxicity did not show these typical FLIO findings.

\section{Anterior segment imaging}

The anterior segment consists of the cornea, iris, suspensory ligaments, trabecular meshwork, lens, and the surrounding aqueous fluid. Only a few studies have investigated the FLTs of the anterior segment of the eye, and most of these focus on properties of the lens. As the human lens ages, post-translational modifications of polypeptides occur, including a variety of photo-oxidative changes that specifically change the tryptophan and non-tryptophan peptides within the crystalline lens [78, 79]. These biochemical changes affect protein structure within the lens, subsequently affecting its intrinsic fluorescence properties. Although FLIO uses a confocal system, FLTs from the retina can be influenced by the lens. It has been shown in several studies that cataracts of the eye lead to a general prolongation of FLIO lifetimes [80, 81]. FLIO lifetimes in healthy eyes had an age-related prolongation [11, 13]. While the LSC showed significant age-specific fluorescence lifetime changes from birth on, the SSC showed significant age-specific changes only after age 35 , presumed to be caused by the lens. One study conducted by Hammer and coworkers analysed the lenses of 32 pre-operative cataract patients. In addition, patients with preexisting ophthalmologic diseases known to influence FLIO results were excluded from the study [79]. Patients' FLIO images were compared before and after surgery, and an overestimation (prolongation) of FLIO lifetimes by $173 \%$ (320 ps) in the SSC, and 48\% (100 ps) in the LSC was found [79]. While the LSC is less affected by lens changes, lens-related SSC prolongation presents challenges to produce reliable FLIO images. Similarly, Klemm et al. [80] compared autofluorescence intensity to mean fluorescence lifetimes when examining patients before and after cataract surgery. Figure 7 shows this impact by depicting the anterior segment before and after cataract surgery. Crystalline lens 
Fig. 6 FLIO in

hydroxychloroquine toxicity.

FAF intensity and FLIO lifetime

images from an eye with

hydroxychloroquine toxicity.

Short (SSC, 498-560 nm) and

long (LSC, $560-720 \mathrm{~nm}$ )

spectral channels are shown.

\section{FLIO Lifetime FAF Intensity}

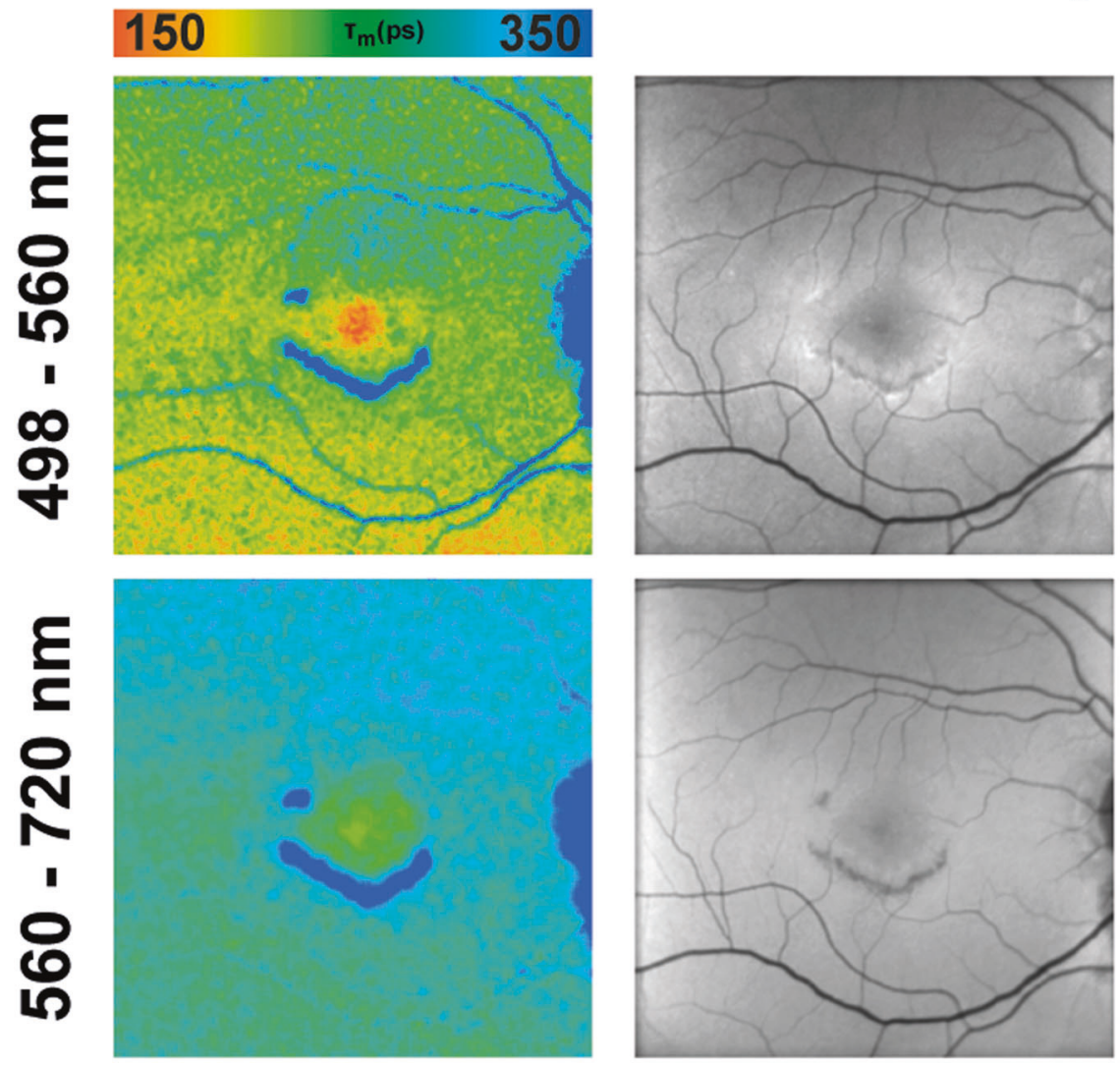

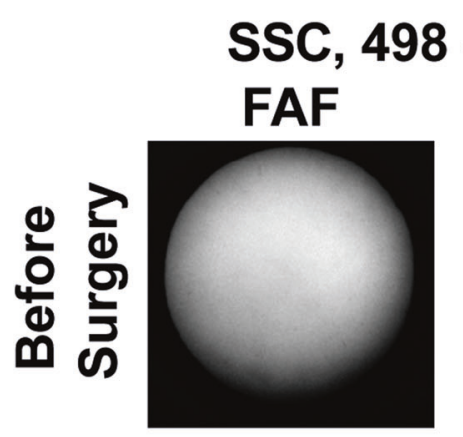

- $560 \mathrm{~nm}$

FLIO
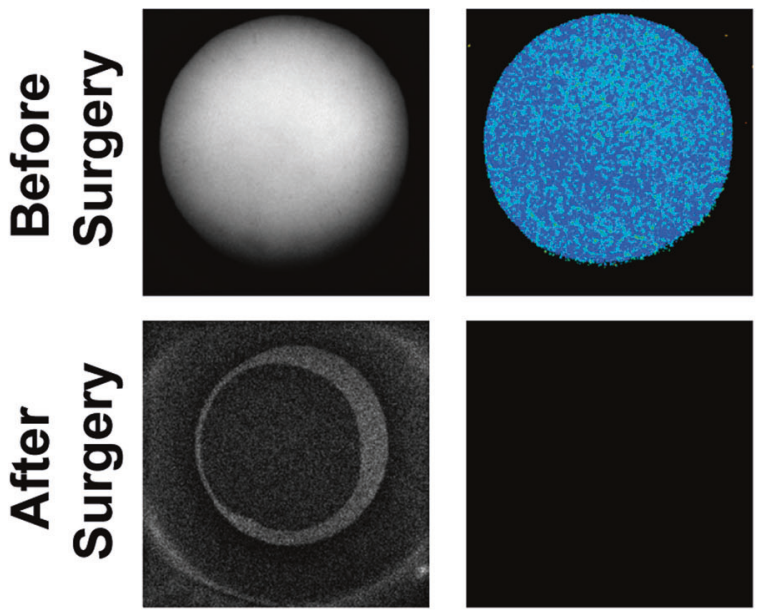

LSC, 560 - 720 nm FAF
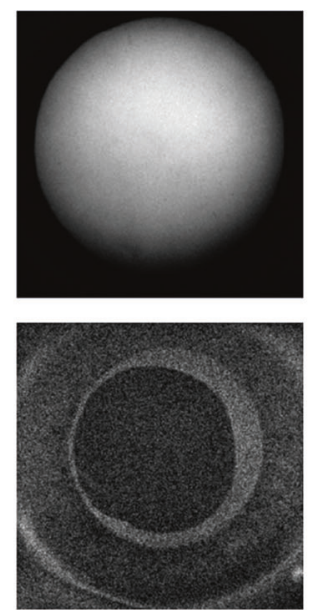

FLIO
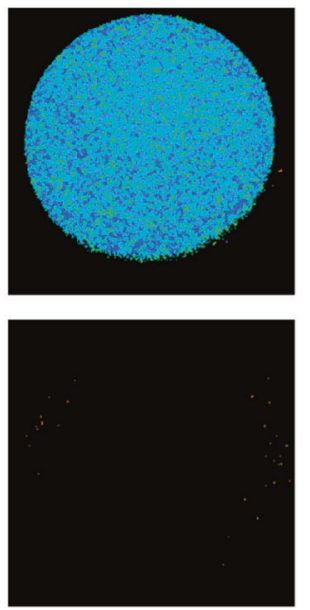

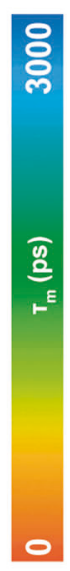

Fig. 7 FLIO in the anterior segment. FAF intensity and FLIO lifetime images of the anterior segment before (top row) and after (bottom row) cataract surgery. Short (SSC, 498-560 nm) and long (LSC, 560-720 nm) spectral channels are shown.

fluorescence in cataract patients reduced the signal-to-noise ratio in FAF intensity imaging, while increasing the FLTs [80]. These findings not only highlight a limitation of the current system but also the importance of finding a solution to minimize the crystalline lens interference in FLIO imaging and image analysis. Multiple approaches have been 
investigated to minimize this effect in the fitting process, and further research is needed to truly eliminate the lens fluorescence from retinal FLIO images [12, 80, 81].

\section{Glaucoma}

Glaucoma has been investigated with FLIO imaging to a limited extent. Only one Letter to the Editor can be found where 43 patients with glaucoma were investigated with an experimental FLIO device [82]. These measurements were compared to those of 54 healthy controls to determine whether FLIO could show the glaucomatous degeneration of retinal ganglion cells. This study was done on patients who received glaucoma profiling at the same time, which required multiple applications of fluorescein drops to the eye during the day for Goldmann applanation tonometry. Unfortunately, fluorescence from fluorescein can overwhelm FLIO signal originating from ocular tissues, an effect that can last up to 24 $\mathrm{h}$. Therefore, it is not surprising that no significant differences were found, and this study should be repeated without concurrent use of fluorescein drops.

\section{Alzheimer disease}

Alzheimer disease (AD) is a progressive neurodegenerative disease that affects an individual's cognitive abilities and memory processes, which is associated with the deposition of amyloid beta $(\mathrm{A} \beta)$ plaques and neurofibrillary tangles of hyperphosphorylated tau in the brain [83]. The close association of the brain and optic nerve provides a window into understanding neurological diseases and their pathologic associations. Previous studies have shown that AD manifests in ocular structures such as the retinal and optic nerve tissues, which can result in retinal degeneration, retinal nerve fibre layer thinning, reduced choroidal thickness, and $\mathrm{A} \beta$ deposition [84, 85]. Novel biomarkers for disease detection and pathology, such as FLIO, have been of great interest in understanding disease onset, localization, and progression of $\mathrm{AD}$.

A pilot study conducted by Jentsch et al. [86] found that changes in FLIO parameters were dependent on the severity of $\mathrm{AD}$. When examining the second component of fluorescence decay (parameters $\tau_{2}, \alpha_{2}, \mathrm{Q}_{2}$ ) in a macula-centred $30^{\circ}$ field, the LSC showed a strong correlation between increasing $\mathrm{Q}_{2}$ and tau and phosphorylated tau proteins ( $r=$ 0.906 and 0.919 , respectively) [86]. These findings indicate that FLIO may be useful as a potential biomarker for ADrelated disease pathology in the neuronal retina. A second pilot study conducted by Sadda and coworkers investigated the correlations between ganglion cell and inner plexiform layer thickness (GCIPL) and A $\beta$ and tau levels with fluorescence lifetime parameters. They reported a strong correlation of fluorescence lifetimes [87]. Prolongation of fluorescence lifetimes in the LSC in association with GCIPL and $\mathrm{A} \beta$ deposition may detect pre-clinical $\mathrm{AD}$.

\section{Outlook and perspectives}

FLIO imaging holds the potential to diagnose, detect, and monitor the progression of a variety of retinal diseases. While this novel technology is currently available at only a few academic research centres, results have been shown to be reproducible at each site. Within our perspectives and future outlook, we would like to draw attention to four retinal conditions; AMD, Stargardt disease, hydroxychloroquine toxicity, and MacTel, for which we found evidence that FLIO may be very useful.

FLIO detects changes consistent with AMD even before typical hallmark signs such as drusen appear, and a consistent ring-shaped pattern of prolonged lifetimes can be found. These FLIO findings may indicate signs of evolving disease. FLIO has also been useful in distinguishing between ambiguous cases of AMD and other retinal diseases. Importantly, Stargardt disease and AMD may clinically appear similar but have very different manifestations in FLIO. FLIO lifetimes in Stargardt disease are not generally prolonged, and the ringshaped pattern is not found in eyes with Stargardt disease. However, the largest benefit for the use of FLIO in Stargardt disease is the detection of early disease-related changes; flecks can be detected about a year prior to being visible in other imaging modalities. Early findings may help determine not only which patients are susceptible to progression, but they can also guide patient selection for clinical trials. Early disease-related changes have also been shown in other inherited retinal diseases such as MacTel. With current clinical trials to treat and slow MacTel under investigation, FLIO may be helpful in identifying patients that are appropriate for treatment even before they experience vision loss. Finally, systemic drugs such as hydroxychloroquine have shown specific FLIO lifetime patterns, even in patients with early retinal toxicity. FLIO may be a quicker and easier alternative than a multifocal ERG for detecting these changes.

It is possible that FLIO will show large benefits for diseases that have not yet been investigated with this device. It would not be surprising to us if future research found additional benefits of FLIO in other retinal diseases and even anterior segment conditions. Some preliminary studies have already found significant changes in patients with diabetes and $\mathrm{AD}$, both of which need further exploration. The unexplored potential of FLIO is exciting and promising. However, it is important to overcome and address some technical pitfalls that may make the broad application of FLIO more difficult.

In the context of pitfalls, we would like to highlight that the current analysis software still needs significant improvement. 
While it is easy to quickly train an inexperienced researcher the technical aspects of FLIO, it is important to be mindful of three major components: proper illumination of the $30^{\circ}$ macular field, precise focus of peri-foveal blood vessels, as well as sufficient photon counts of at least 1000 photons in each spectral channel. The imaging time is typically 2 min for each eye, but it can take upwards of 3 min in patients with advanced macular atrophy. We recognize that it may be beneficial to obtain higher photon counts of up to 1500 photons in the macula of patients with early cataracts, as this can improve fitting parameters of the retina.

Fitting the FLIO data to obtain the final images poses a major challenge. The software used to fit the raw data is a spin-off from one that has been traditionally used in cell culture analysis. Although this software, SPCImage, is very advanced for cell cultures, it lacks some features that may be useful in clinical analysis. The rather complex and timeconsuming software may seem overwhelming to clinicians who rely on more straightforward and efficient programmes to generate clinical images. Furthermore, the software is not well equipped for the clinical analysis of lifetimes, such as the ability to obtain the mean from standardized areas such as ETDRS grid. Instead, secondary programmes such as FLIMX and FLIO-Reader are used to accomplish this. Raw data from SPCImage must first be exported and then imported into the secondary programme to obtain mean lifetimes over certain areas, which is not sustainable or practical in a clinical setting. In addition, one must be careful not to create fitting errors that may be falsely interpreted as disease-related changes. Overall, we see the fitting process as very complex and believe that addressing the image analysis will be key in establishing the universality of FLIO in academic centres.

Aside from these pitfalls, we regard FLIO as a modality that holds promising potential in the field of retinal imaging, in addition to its insight in ambiguous ophthalmic and systemic diseases. More research will be necessary to truly establish this device in the clinical arena. Therefore, we would like to stress the importance of ongoing research and expansion of FLIO technology as we look forward to the era when FLIO can become more widely accessible.

Funding NIH grants EY11600 and EY14800; Lowy Medical Research Institute, Research to Prevent Blindness.

\section{Compliance with ethical standards}

Conflict of interest LS, ASV, PSB: medical consultants for Tesseract Health, Inc. All: The FLIO device was provided by Heidelberg Engineering at no cost. No financial support was received from Heidelberg Engineering.

Publisher's note Springer Nature remains neutral with regard to jurisdictional claims in published maps and institutional affiliations.

\section{References}

1. Lakowicz JR. Principles of fluorescence spectroscopy. New York: Springer; 2007.

2. Marcu L. Fluorescence lifetime techniques in medical applications. Ann Biomed Eng. 2012;40:304-31.

3. Lakowicz JR, Szmacinski H, Nowaczyk K, Johnson ML. Fluorescence lifetime imaging of free and protein-bound NADH. Proc Natl Acad Sci USA. 1992;89:1271-5.

4. Schweitzer D, Kolb A, Hammer M, Anders R. Time-correlated measurement of autofluorescence. A method to detect metabolic changes in the fundus. Ophthalmologe. 2002;99:774-9.

5. Schweitzer D, Schenke S, Hammer M, Schweitzer F, Jentsch S, Birckner E, et al. Towards metabolic mapping of the human retina. Microsc Res Tech. 2007;70:410-9.

6. Becker W. The bh TCSPC handbook. 6th ed. Berlin: Becker \& Hickl GmbH; 2014.

7. Sauer L, Schweitzer D, Ramm L, Augsten R, Hammer M, Peters S. Impact of macular pigment on fundus autofluorescence lifetimes. Investig Ophthalmol Vis Sci. 2015;56:4668-79.

8. ANSI. American National Standard for the Safe Use of Lasers. Orlando, FL: Laser Institute of America; 2000.

9. Delori FC, Webb RH, Sliney DH, American National Standards I. Maximum permissible exposures for ocular safety (ANSI 2000), with emphasis on ophthalmic devices. J Opt Soc Am A Opt Image Sci Vis. 2007;24:1250-65.

10. Sauer L, Andersen KM, Li B, Gensure RH, Hammer M, Bernstein PS. Fluorescence lifetime imaging ophthalmoscopy (FLIO) of macular pigment. Investig Ophthalmol Vis Sci. 2018;59:3094-103.

11. Sauer L, Vitale AS, Milliken CM, Modersitzki NK, Blount D, Bernstein PS. Autofluorescence lifetimes measured with fluorescence lifetime imaging ophthalmoscopy (FLIO) are affected by age, but not by pigmentation or gender. Transl Vis Sci Technol. 2020;9:2.

12. Klemm M, Dietzel A, Haueisen J, Nagel E, Hammer M, Schweitzer D. Repeatability of autofluorescence lifetime imaging at the human fundus in healthy volunteers. Curr Eye Res. 2013;38:793-801.

13. Dysli C, Quellec G, Abegg M, Menke MN, Wolf-Schnurrbusch U, Kowal J, et al. Quantitative analysis of fluorescence lifetime measurements of the macula using the fluorescence lifetime imaging ophthalmoscope in healthy subjects. Investig Ophthalmol Vis Sci. 2014;55:2106-13.

14. Kwon S, Borrelli E, Fan W, Ebraheem A, Marion KM, Sadda SR. Repeatability of fluorescence lifetime imaging ophthalmoscopy in normal subjects with mydriasis. Transl Vis Sci Technol. 2019;8:15.

15. Klemm M, Sauer L, Klee S, Link D, Peters S, Hammer M, et al. Bleaching effects and fluorescence lifetime imaging ophthalmoscopy. Biomed Opt Express. 2019;10:1446-61.

16. Hammer M, Konigsdorffer E, Liebermann C, Framme C, Schuch G, Schweitzer D, et al. Ocular fundus auto-fluorescence observations at different wavelengths in patients with age-related macular degeneration and diabetic retinopathy. Graefes Arch Clin Exp Ophthalmol. 2008;246:105-14.

17. Sauer L, Peters S, Schmidt J, Schweitzer D, Klemm M, Ramm L, et al. Monitoring macular pigment changes in macular holes using fluorescence lifetime imaging ophthalmoscopy. Acta Ophthalmol. 2017;95:481-92.

18. Ermakov IV, McClane RW, Gellermann W, Bernstein PS. Resonant Raman detection of macular pigment levels in the living human retina. Opt Lett. 2001;26:202-4.

19. Jaggi DSY, Dysli C, Wolf S, Zinkernagel M. Fluorescence lifetime imaging ophthalmoscopy and the influence of oral lutein supplementation on macular pigment: a preliminary study report. Paris: EURetina; 2019. 
20. Greenberg JP, Duncker T, Woods RL, Smith RT, Sparrow JR, Delori FC. Quantitative fundus autofluorescence in healthy eyes. Investig Ophthalmol Vis Sci. 2013;54:5684-93.

21. Friedman DS, O'Colmain BJ, Munoz B, Tomany SC, McCarty C, de Jong PT, et al. Prevalence of age-related macular degeneration in the United States. Arch Ophthalmol. 2004;122:564-72.

22. Gehrs KM, Anderson DH, Johnson LV, Hageman GS. Age-related macular degeneration-emerging pathogenetic and therapeutic concepts. Ann Med. 2006;38:450-71.

23. Klein R, Cruickshanks KJ, Nash SD, Krantz EM, Nieto FJ, Huang $\mathrm{GH}$, et al. The prevalence of age-related macular degeneration and associated risk factors. Arch Ophthalmol. 2010;128:750-8.

24. Ferris FL 3rd, Wilkinson CP, Bird A, Chakravarthy U, Chew E, Csaky K, et al. Clinical classification of age-related macular degeneration. Ophthalmology. 2013;120:844-51.

25. Age-Related Eye Disease Study 2 Research Group, Chew EY, Clemons TE, Sangiovanni JP, et al. Secondary analyses of the effects of lutein/zeaxanthin on age-related macular degeneration progression: AREDS2 report No. 3. JAMA Ophthalmol. 2014;132:142-9.

26. Sauer L, Gensure RH, Andersen KM, Kreilkamp L, Hageman GS, Hammer M, et al. Patterns of fundus autofluorescence lifetimes in eyes of individuals with nonexudative age-related macular degeneration. Investig Ophthalmol Vis Sci. 2018;59:AMD65-77.

27. Dysli C, Fink R, Wolf S, Zinkernagel MS. Fluorescence lifetimes of drusen in age-related macular degeneration. Investig Ophthalmol Vis Sci. 2017;58:4856-62.

28. Dysli C, Wolf S, Zinkernagel MS. Autofluorescence lifetimes in geographic atrophy in patients with age-related macular degeneration. Investig Ophthalmol Vis Sci. 2016;57:2479-87.

29. Sauer L, Klemm M, Peters S, Schweitzer D, Schmidt J, Kreilkamp $\mathrm{L}$, et al. Monitoring foveal sparing in geographic atrophy with fluorescence lifetime imaging ophthalmoscopy-a novel approach. Acta Ophthalmol. 2018;96:257-66.

30. Hageman GS, Mullins RF. Molecular composition of drusen as related to substructural phenotype. Mol Vis. 1999;5:28.

31. Pepple K, Mruthyunjaya P. Retinal pigment epithelial detachments in age-related macular degeneration: classification and therapeutic options. Semin Ophthalmol. 2011;26:198-208.

32. Sauer L, Komanski CB, Vitale AS, Hansen ED, Bernstein PS. Fluorescence lifetime imaging ophthalmoscopy (FLIO) in eyes with pigment epithelial detachments due to age-related macular degeneration. Investig Ophthalmol Vis Sci. 2019;60:3054-63.

33. Schweitzer D, Gaillard ER, Dillon J, Mullins RF, Russell S, Hoffmann B, et al. Time-resolved autofluorescence imaging of human donor retina tissue from donors with significant extramacular drusen. Investig Ophthalmol Vis Sci. 2012;53:3376-86.

34. Schweitzer D, Quick S, Schenke S, Klemm M, Gehlert S, Hammer $\mathrm{M}$, et al. Comparison of parameters of time-resolved autofluorescence between healthy subjects and patients suffering from early AMD. Ophthalmologe. 2009;106:714-22.

35. Eisma JH, Dulle JE, Fort PE. Current knowledge on diabetic retinopathy from human donor tissues. World J Diabetes. 2015;6: 312-20.

36. Simo R, Hernandez C. Novel approaches for treating diabetic retinopathy based on recent pathogenic evidence. Prog Retin Eye Res. 2015;48:160-80.

37. Zhang X, Zeng H, Bao S, Wang N, Gillies MC. Diabetic macular edema: new concepts in patho-physiology and treatment. Cell Biosci. 2014;4:27.

38. Guerin-Dubourg A, Cournot M, Planesse C, Debussche X, Meilhac O, Rondeau P, et al. Association between fluorescent advanced glycation end-products and vascular complications in type 2 diabetic patients. Biomed Res Int. 2017;2017:7989180.

39. Schmidt J, Peters S, Sauer L, Schweitzer D, Klemm M, Augsten R, et al. Fundus autofluorescence lifetimes are increased in nonproliferative diabetic retinopathy. Acta Ophthalmol. 2017;95:33-40.
40. Schweitzer D, Deutsch L, Klemm M, Jentsch S, Hammer M, Peters S, et al. Fluorescence lifetime imaging ophthalmoscopy in type 2 diabetic patients who have no signs of diabetic retinopathy. J Biomed Opt. 2015;20:61106.

41. Schweitzer D. Metabolic mapping. In: Holz F, Spaide R, editors. Medical retina. Essentials in ophthalmology. Berlin: Springer Berlin Heidelberg; 2010. p. 107-23.

42. Schweitzer D, Quick S, Klemm M, Hammer M, Jentsch S, Dawczynski J. Time-resolved autofluorescence in retinal vascular occlusions. Ophthalmologe. 2010;107:1145-52.

43. Hammer M, Sauer L, Klemm M, Peters S, Schultz R, Haueisen J. Fundus autofluorescence beyond lipofuscin: lesson learned from ex vivo fluorescence lifetime imaging in porcine eyes. Biomed Opt Express. 2018;9:3078-91.

44. Abulrob A, Brunette E, Slinn J, Baumann E, Stanimirovic D. Dynamic analysis of the blood-brain barrier disruption in experimental stroke using time domain in vivo fluorescence imaging. Mol Imaging. 2008;7:7290.2008.00025.

45. Hamasaki DI, Kroll AJ. Experimental central retinal artery occlusion: an electrophysiological study. Arch Ophthalmol. 1968;80:243-8.

46. Semeraro F, Morescalchi F, Russo A, Gambicorti E, Pilotto A, Parmeggiani F, et al. Central serous chorioretinopathy: pathogenesis and management. Clin Ophthalmol. 2019;13:2341-52.

47. Iyer PG, Schwartz SG, Russell JF, Flynn HW. Central serous chorioretinopathy: multimodal imaging and management options. Case Rep Ophthalmol. 2020;2020:8890404.

48. Dysli C, Berger L, Wolf S, Zinkernagel MS. Fundus autofluorescence lifetimes and central serous chorioretinopathy. Retina. 2017;37:2151-61.

49. Charbel Issa P, Gillies MC, Chew EY, Bird AC, Heeren TF, Peto $\mathrm{T}$, et al. Macular telangiectasia type 2. Prog Retin Eye Res. 2013;34:49-77.

50. Chew EY, Clemons TE, Peto T, Sallo FB, Ingerman A, Tao W, et al. Ciliary neurotrophic factor for macular telangiectasia type 2: results from a phase 1 safety trial. Am J Ophthalmol. 2015;159:659-66 e1.

51. Gantner ML, Eade K, Wallace M, Handzlik MK, Fallon R, Trombley J, et al. Serine and lipid metabolism in macular disease and peripheral neuropathy. N Engl J Med. 2019;381:1422-33.

52. Sauer L, Gensure RH, Hammer M, Bernstein PS. Fluorescence lifetime imaging ophthalmoscopy: a novel way to assess macular telangiectasia type 2. Ophthalmol Retina 2018;2:587-98.

53. Sauer L, Vitale AS, Andersen KM, Hart B, Bernstein PS. Fluorescence lifetime imaging ophthalmoscopy (FLIO) patterns in clinically unaffected children of macular telangiectasia type 2 (MacTel) patients. Retina. 2020;40:695-704.

54. Solberg Y, Dysli C, Wolf S, Zinkernagel MS. Fluorescence lifetime patterns in macular telangiectasia type 2. Retina. 2020;40:99-108.

55. Michaelides M, Hunt DM, Moore AT. The genetics of inherited macular dystrophies. J Med Genet. 2003;40:641-50.

56. Stone EM, Andorf JL, Whitmore SS, DeLuca AP, Giacalone JC, Streb LM, et al. Clinically focused molecular investigation of 1000 consecutive families with inherited retinal disease. Ophthalmology. 2017;124:1314-31.

57. Allikmets R, Singh N, Sun H, Shroyer NF, Hutchinson A, Chidambaram A, et al. A photoreceptor cell-specific ATP-binding transporter gene (ABCR) is mutated in recessive Stargardt macular dystrophy. Nat Genet. 1997;15:236-46.

58. Boyer NP, Higbee D, Currin MB, Blakeley LR, Chen C, Ablonczy $\mathrm{Z}$, et al. Lipofuscin and N-retinylidene-Nretinylethanolamine (A2E) accumulate in retinal pigment epithelium in absence of light exposure: their origin is 11-cis-retinal. $\mathrm{J}$ Biol Chem. 2012;287:22276-86.

59. Zhang N, Tsybovsky Y, Kolesnikov AV, Rozanowska M, Swider M, Schwartz SB, et al. Protein misfolding and the pathogenesis of ABCA4-associated retinal degenerations. Hum Mol Genet. 2015; 24:3220-37. 
60. Cideciyan AV, Aleman TS, Swider M, Schwartz SB, Steinberg $\mathrm{JD}$, Brucker AJ, et al. Mutations in ABCA4 result in accumulation of lipofuscin before slowing of the retinoid cycle: a reappraisal of the human disease sequence. Hum Mol Genet. 2004;13:525-34.

61. Dysli C, Wolf S, Hatz K, Zinkernagel MS. Fluorescence lifetime imaging in Stargardt disease: potential marker for disease progression. Investig Ophthalmol Vis Sci. 2016;57:832-41.

62. Solberg Y, Dysli C, Escher P, Berger L, Wolf S, Zinkernagel MS. Retinal flecks in Stargardt disease reveal characteristic fluorescence lifetime transition over time. Retina. 2019;39:879-88.

63. Ablonczy Z, Gutierrez DB, Grey AC, Schey KL, Crouch RK. Molecule-specific imaging and quantitation of A2E in the RPE. Adv Exp Med Biol. 2012;723:75-81.

64. Sears AE, Bernstein PS, Cideciyan AV, Hoyng C, Charbel Issa P, Palczewski K, et al. Towards treatment of Stargardt disease: workshop organized and sponsored by the foundation fighting blindness. Transl Vis Sci Technol. 2017;6:6.

65. Mitsios A, Dubis AM, Moosajee M. Choroideremia: from genetic and clinical phenotyping to gene therapy and future treatments. Ther Adv Ophthalmol. 2018;10:2515841418817490.

66. Vitale AS, Sauer L, Modersitzki NK, Bernstein PS. Fluorescence lifetime imaging ophthalmoscopy (FLIO) in patients with choroideremia. Transl Vis Sci Technol 2020;9:33.

67. Pagon RA. Retinitis pigmentosa. Surv Ophthalmol. 1988;33:137-77.

68. Dysli C, Schurch K, Pascal E, Wolf S, Zinkernagel MS. Fundus autofluorescence lifetime patterns in retinitis pigmentosa. Investig Ophthalmol Vis Sci. 2018;59:1769-78.

69. Andersen KM, Sauer L, Gensure RH, Hammer M, Bernstein PS. Characterization of retinitis pigmentosa using fluorescence lifetime imaging ophthalmoscopy (FLIO). Transl Vis Sci Technol. 2018;7:20.

70. Jackson TL, Nicod E, Simpson A, Angelis A, Grimaccia F, Kanavos P. Symptomatic vitreomacular adhesion. Retina. 2013;33:1503-11.

71. Jaggi D, Solberg Y, Dysli C, Ebneter A, Wolf S, Zinkernagel MS. Fluorescence lifetime imaging ophthalmoscopy: findings after surgical reattachment of macula-off rhegmatogenous retinal detachment. Retina. 2020;40:1929-37.

72. Tett SE, Cutler DJ, Day RO, Brown KF. Bioavailability of hydroxychloroquine tablets in healthy volunteers. Br J Clin Pharmacol. 1989;27:771-9.

73. de Sisternes L, Hu J, Rubin DL, Marmor MF. Localization of damage in progressive hydroxychloroquine retinopathy on and off the drug: inner versus outer retina, parafovea versus peripheral fovea. Investig Ophthalmol Vis Sci. 2015;56:3415-26.

74. Marmor MF, Kellner U, Lai TY, Melles RB, Mieler WF.American Academy of Ophthalmology Recommendations on screening for chloroquine and hydroxychloroquine retinopathy (2016 Revision). Ophthalmology. 2016;123:1386-94.
75. Jorge A, Ung C, Young LH, Melles RB, Choi HK. Hydroxychloroquine retinopathy-implications of research advances for rheumatology care. Nat Rev Rheumatol. 2018;14:693-703.

76. Sauer LC, Calvo CM, Vitale, AS, Henrie, N, Milliken, CM, Bernstein, PS. Imaging of hydroxychloroquine toxicity with fluorescence lifetime imaging ophthalmoscopy (FLIO). Ophthalmol Retina. 2019;3:814-25.

77. Solberg Y, Dysli C, Moller B, Wolf S, Zinkernagel MS. Fluorescence lifetimes in patients with hydroxychloroquine retinopathy. Investig Ophthalmol Vis Sci. 2019;60:2165-72.

78. Gakamsky A, Duncan RR, Howarth NM, Dhillon B, ButtenschN KK, Daly DJ, et al. Tryptophan and non-tryptophan fluorescence of the eye lens proteins provides diagnostics of cataract at the molecular level. Sci Rep. 2017;7:40375.

79. Brauer JL, Schultz R, Klemm M, Hammer M. Influence of lens fluorescence on fluorescence lifetime imaging ophthalmoscopy (FLIO) fundus imaging and strategies for its compensation. Transl Vis Sci Technol. 2020;9:13.

80. Klemm M, Blum J, Link D, Hammer M, Haueisen J, Schweitzer D. Combination of confocal principle and aperture stop separation improves suppression of crystalline lens fluorescence in an eye model. Biomed Opt Express. 2016;7:3198-210.

81. Schweitzer D, Hammer M, Schweitzer F. Limits of the confocal laser-scanning technique in measurements of time-resolved autofluorescence of the ocular fundus. Biomed Tech. 2005; 50:263-7.

82. Ramm L, Jentsch S, Augsten R, Hammer M. Fluorescence lifetime imaging ophthalmoscopy in glaucoma. Graefe's Arch Clin Exp Ophthalmol. 2014;252:2025-6.

83. Weller J, Budson A. Current understanding of Alzheimer's disease diagnosis and treatment. F1000Res. 2018;7:F1000 Faculty Rev-1161.

84. Bambo MP, Garcia-Martin E, Pinilla J, Herrero R, Satue M, Otin $S$, et al. Detection of retinal nerve fiber layer degeneration in patients with Alzheimer's disease using optical coherence tomography: searching new biomarkers. Acta Ophthalmol. 2014;92: e581-2.

85. Javaid FZ, Brenton J, Guo L, Cordeiro MF. Visual and ocular manifestations of Alzheimer's disease and their use as biomarkers for diagnosis and progression. Front Neurol. 2016;7:55.

86. Jentsch S, Schweitzer D, Schmidtke KU, Peters S, Dawczynski J, Bär KJ, et al. Retinal fluorescence lifetime imaging ophthalmoscopy measures depend on the severity of Alzheimer's disease. Acta Ophthalmol. 2015;93:e241-7.

87. Kwon S, Fang W, Borreli E, Ebraheem A, Marion K, Katayama $\mathrm{Y}$, et al. Fluorescence lifetime imaging ophthalmoscopy in early Alzheimer's disease. Honolulu, Hawaii, USA: ARVO Annual Meeting; 2018. 